\title{
The effect of maternal diabetes on the synthesis and secretion of phosphatidylcholine in fetal and maternal rat lungs in vitro
}

\author{
M.S. Nijjar ${ }^{1,2}$, B.S. Khangura ${ }^{1 *}$ and L. I. Juravsky ${ }^{1 * *}$ \\ ${ }^{1}$ Department of Pathology, Faculty of Medicine, University of Manitoba, Winnipeg; ${ }^{2}$ Alvin Buckwold Centre, Department of Pediatrics, \\ University of Saskatchewan, Saskatoon, Saskatchewan, Canada
}

\begin{abstract}
Summary. Incorporation of (methyl- ${ }^{14} \mathrm{C}$ ) choline into phosphatidylcholine and the release of prelabelled phosphatidylcholine was investigated in vitro using lung slices from pregnant rats and their offspring near term. Tissue from normal, diabetic and insulin-treated diabetic pregnant rats and their offspring was utilized to assess the effects of maternal diabetes on fetal lung maturation. The results show that the synthesis of phosphatidylcholine in fetal/newborn lungs through the cytidine $5^{\prime}$-diphosphocholine pathway was not affected by maternal diabetes. However, secretion of phosphatidylcholine from the lungs of fetuses of diabetic mothers was very much suppressed one day after parturition. Insulin treatment of the diabetic pregnant rats restored secretion of phosphatidylcholine from the fetal/newborn lungs to control values. These re-
\end{abstract}

sults suggest that an impaired secretion of phosphatidylcholine from the lungs of fetuses of diabetic mothers may be partly responsible for the higher incidence of respiratory distress syndrome among children of diabetic mothers. The results also revealed some correlation between the secretion of phosphatidylcholine from maternal lungs, maternal serum phospholipids and synthesis of phosphatidylcholine by fetal lungs during late gestation, suggesting a possible relationship between maternal phospholipid metabolism and fetal lung maturation.

Key words: Maternal diabetes, synthesis and secretion, phosphatidylcholine.
Certain phospholipids are important for lung function (respiration) particularly at the time of birth. Lungs of children deficient in surfactant phospholipids tend to collapse during expiration resulting in the death of a newborn, the syndrome commonly known as respiratory distress syndrome or hyaline membrane disease [1]. The children of diabetic mothers show higher incidence of respiratory distress syndrome. The precise biochemical aetiology of respiratory distress syndrome in diabetic children is uncertain although a deficiency of surfactant in their lungs has been implicated [2]. A defective secretion of surfactant from the alveolar type II cells into the alveolar space could contribute to surfactant deficiency. However, this aspect of surfactant metabolism has not been investigated fully.

Synthesis of surfactant phospholipids appears to be dependent on metabolism of glucose [3-5] and glycogen [6-9] as they provide dihydroxyacetone phosphate and $\alpha$-glyceraldehyde phosphate, which are precursors of phospholipid synthesis in lungs $[6,9,10]$. In maternal diabetes, the fetus is exposed to hyperglycaemia and hyperinsulinaemia both of which inhibit glycogenolysis

\footnotetext{
Present address: * Department of Crop Science, University of Saskatchewan, Saskatoon, Saskatchewan Canada, S7N 0W0

** Faculty of Medicine, University of Manitoba, Winnipeg, Canada R3E 0W3
}

and reduce the formation of precursors for synthesis of surfactant phospholipids [9,11-13]. While some investigators have reported reduced synthesis of surfactant phospholipids in lungs of fetuses of diabetic mothers $[14,15]$ others have not observed any significant changes in the phospholipid synthesis $[16,17]$. However, surfactant deficiency in the alveolar space could result from some defect in the process of surfactant secretion from the alveolar type II cells [37].

Our previous studies with adult rat lungs have shown that streptozotocin-induced diabetes altered the regulation of adenylate cyclase [18] and cyclic AMP phosphodiesterase [19] by the intracellular modulators, such as the lung activator [20] and calmodulin, respectively. Relative activities of these enzymes appear to determine the tissue levels of cyclic AMP $[21,22]$ which appears to play a significant role in pulmonary development and functions [21-25]. In the present study, the effects of maternal diabetes on the synthesis and secretion of phosphatidylcholine (PC), a major component of surfactant phospholipids, in fetal lungs during late gestation and early neonatal periods of development (perinatal life) were investigated. The effects of diabetes on the maternal lung phospholipid metabolism was also assessed simultaneously under identical conditions in an attempt to ascertain whether maternal phospholipid metabolism was somehow related to fetal lung develop- 
ment. Some of these data have been published previously [26].

\section{Materials and methods}

\section{Biochemical reagents}

Most biochemicals used in this study were of analytical grade and purchased from either Sigma Chemicals, St. Louis, Missouri, USA or Fisher Scientific, Winnipeg, Manitoba, Canada. (Methyl- ${ }^{14} \mathrm{C}$ )choline was purchased from New England Nuclear, Chicago, Illinois, USA.

\section{Animal breeding and maintenance}

Sprague-Dawley rats, weighing $250-300 \mathrm{~g}$, were purchased from Charles River Breeding Farms, Montreal, Quebec. Five female rats were placed in a cage with two male rats overnight and examined for sperm cells by light microscopy next morning. Those female rats containing sperm cells in their vagina were considered pregnant and day 1 of gestation was assigned to their fetuses. Full term was 22 days of gestation. These animals were provided with water and pellet chow ad libitum and $12 \mathrm{~h}$ light and dark cycle was maintained in the animal quarters.

\section{Streptozotocin-induced diabetes and treatment with insulin}

On day 9 of pregnancy, a group of 25 rats was injected via the tail vein with streptozotocin $(65 \mathrm{mg} / \mathrm{kg}$ body weight) freshly prepared in $0.1 \mathrm{~mol} / 1$ citrate buffer $(\mathrm{pH} 4.6)$ on the day of use. Animals were made diabetic on day 9 of pregnancy to allow exposure of fetuses to hyperglycaemia and hyperinsulinaemia for at least one week to assess the effects of maternal diabetes on surfactant synthesis and secretion.

The control group contained 10 age-matched pregnant rats who received an equal volume of the citrate buffer by a similar route. Ten pregnant diabetic rats were treated daily with 6 units of protamine zinc insulin (Connaught Laboratories, Willowdale, Ontario) starting on day 16 of gestation, whereas the remaining 15 diabetic rats were treated with an equal volume of $150 \mathrm{mmol} / 1 \mathrm{NaCl}$ solution. Insulin was administered to diabetic pregnant rats on day 16 of pregnancy to restore normoglycaemia and to ascertain whether the effects of maternal diabetes on surfactant synthesis and secretion in fetal lungs could be restored to normal values in control fetuses.

Glycosuria was confirmed in all the rats treated with streptozotocin within $24 \mathrm{~h}$ of injection using Tes-Tape impregnated with the enzymes glucose oxidase and peroxidase and an oxidizable substrate orthotoluidine (Eli Lilly, Scarborough, Ontario). Glucose intolerance was confirmed $72 \mathrm{~h}$ after the injection with streptozotocin by estimating glucose in sera of diabetic rats by the glucose oxidase method [27].

\section{Tissue preparation for metabolic studies}

At least three rats from the control, diabetic and insulin-treated diabetic groups were sacrificed on days $18,20,21$ of gestation and on days 1 and 2 after parturition. Lungs from the mothers, fetuses and the newborn were excised, cleaned of extraneous tissues and weighed. Only the peripheral portion of lung containing predominently alveolar tissue was used to prepare slices of uniform thickness $\left(1 \mathrm{~mm}^{2}\right)$ cut with the Mcllwain chopper (Brinkman Instruments, Westbury, New York).

\section{Secretion of phosphatidylcholine from rat lungs}

The tissue slices were placed in a medium containing Krebs-Ringer bicarbonate buffer ( $\mathrm{pH} 7.4)$, glucose $(11 \mathrm{mmol} / \mathrm{l})$, bovine serum albu$\min (450 \mu \mathrm{mol} / 1)$ and $\left(\right.$ methyl $\left.-{ }^{14} \mathrm{C}\right)$ choline chloride $(1.0 \mu \mathrm{Ci} ; \mathrm{Sp}$. act.
$50 \mathrm{mCi} / \mathrm{mmol}$ ) in a total volume of $1.0 \mathrm{ml}$. The tubes were incubated in a Dubnoff metabolic shaker (Precision Scientific, Chicago, Illinois) at $37^{\circ} \mathrm{C}$ for $60 \mathrm{~min}$ under $\mathrm{O}_{2} / \mathrm{CO}_{2}(95 \% / 5 \%)$. The reaction was terminated by the addition of ice-cold saline $(150 \mathrm{mmol} / \mathrm{l} \mathrm{NaCl}, 2 \mathrm{ml})$, the medium was withdrawn and the slices washed three times with $2 \mathrm{ml}$ of saline. To examine the release of radiolabelled PC, fresh medium as above was added to the washed prelabelled slices, which were further incubated for 90 min under identical conditions. After incubation, the medium was withdrawn and extracted for lipids by the method of Folch et al. [28]. The control tissue slices were prelabelled with (methyl- ${ }^{14} \mathrm{C}$ ) choline, washed similarly and incubated at $0^{\circ} \mathrm{C}$ during the second incubation to measure non-specific release of labelled PC. The release of radiolabelled $\mathrm{PC}$ from lung slices incubated at $37^{\circ} \mathrm{C}$ was corrected for radioactivity released from the control slices. Under these experimental conditions, secretion of PC from lung slices was linear with time up to 120 min of the second incubation and $50 \mathrm{mg}$ tissue slice/flask.

\section{Synthesis of phosphatidylcholine by rat lungs}

After the first incubation with radioactive choline, lung slices were washed several times with ice-cold saline and extracted for lipids as described above.

The lipid extracts from lung slices were resolved into individual species by a single dimension thin-layer chromatography in a solvent system containing chloroform, methanol and water $(14: 6: 1, \mathrm{vol} / \mathrm{vol})$, and visualized by exposing the dried chromatographic plate to iodine vapour. Silica gel containing the lipids was scraped into counting vials, mixed with $15 \mathrm{ml}$ of scintiverse (Fisher Scientific, Pittsburgh, PA, USA) and the radioactivity measured in a Searle Mark III radiospectrometer (Searle Analytic, des Plaines, Illinois, USA). In some experiments, PC was eluted from the silica gel with a chloroform, methanol, acetic acid and water mixture $(50: 50: 2: 1$, by vol), the extract treated with osmium tetraoxide in carbon tetrachloride and subjected to a single-dimension thin-layer chromatography according to the procedure of Mason et al. [29].

\section{Statistical analysis}

The statistical difference between data from different groups was determined by Student's t-test for unpaired samples [30].

\section{Results}

Streptozotocin-treatment of pregnant rats resulted in significantly elevated maternal serum glucose levels compared with that of the control group of animals (Table 1). Glycosuria was observed in all rats injected with streptozotocin within $24 \mathrm{~h}$ of the injection. The mean fetal serum glucose level also was elevated during late gestation but declined considerably 1 day post-partum. Insulin treatment of the diabetic mothers lowered maternal serum glucose levels to the control values, whereas fetal serum glucose levels were still somewhat higher than those of the control fetuses (Table 1). Newborn serum glucose levels of diabetic mothers returned to the control level 1 day after birth (Table 1). Streptozotocin-induced diabetes in pregnant rats on day 9 of pregnancy altered neither maternal body or lung weights on day 21 of pregnancy nor fetal body and lung weights (Table 2).

The incorporation of (methyl- ${ }^{14} \mathrm{C}$ ) choline into maternal lungs was low on days 18 and 20 of gestation, increased considerably on day 21 , reaching a peak level 
Table 1. Serum glucose concentration in pregnant rats, their fetuses on day 21 of gestation and newborns 1 day after birth

\begin{tabular}{lccl}
\hline & \multicolumn{3}{c}{ Serum glucose concentration (mmol/1) } \\
\cline { 2 - 4 } & Mothers & $\begin{array}{l}\text { Fetuses } \\
(n=25)^{\mathrm{c}}\end{array}$ & $\begin{array}{l}\text { Newborns } \\
(n=23)^{\mathrm{c}}\end{array}$ \\
\hline Control rats $(n=9)^{\mathrm{b}}$ & $6.77 \pm 0.39$ & $2.66 \pm 0.67$ & $4.22 \pm 0.28$ \\
Diabetic rats $(n=8)^{\mathrm{b}}$ & $35.86 \pm 2.22^{\mathrm{a}}$ & $28.14 \pm 1.28^{\mathrm{a}}$ & $6.77 \pm 0.50^{\mathrm{a}}$ \\
Insulin-treated diabetic & $4.44 \pm 0.50$ & $6.94 \pm 0.22^{\mathrm{a}}$ & $2.83 \pm 0.28$ \\
rats $(n=7)^{\mathrm{b}}$ & & & \\
\hline
\end{tabular}

Results expressed as mean \pm SEM for three different experiments.

a Significantly different from the control groups $(p<0.05)$

${ }^{b} n$ refers to total number of pregnant rats used in the control, diabetic and insulin-treated diabetic groups

${ }^{c} n$ refers to number of fetuses and newborns examined for serum glucose from each group of pregnant rats, i.e. control, diabetic and insulin-treated diabetic groups

Table 2. Effect of maternal diabetes on body and lung weights of mother and fetal rats on day 21 of gestation

\begin{tabular}{llllll}
\hline & \multicolumn{2}{l}{ Maternal } & & Fetal & \\
\cline { 2 - 3 } \cline { 5 - 6 } & Body $(\mathrm{g})$ & Lung $(\mathrm{g})$ & & $\begin{array}{l}\text { Body }(\mathrm{g}) \\
(n=35)^{\mathrm{c}}\end{array}$ & $\begin{array}{l}\text { Lung }(\mathrm{g}) \\
(n=30)^{\mathrm{c}}\end{array}$ \\
\hline $\begin{array}{l}\text { Control Rats } \\
(n=9)^{\mathrm{b}}\end{array}$ & $279 \pm 26$ & $1.00 \pm 0.05$ & $3.73 \pm 0.09$ & $0.15 \pm 0.03$ \\
$\begin{array}{l}\text { Diabetic Rats } \\
(n=7)^{\mathrm{b}}\end{array}$ & $234 \pm 24$ & $1.18 \pm 0.17$ & $3.06 \pm 0.10$ & $0.15 \pm 0.01$ \\
$\begin{array}{l}\text { Insulin-treated } \\
\text { diabetic Rats } \\
(n=7)^{\mathrm{b}}\end{array}$ & $344 \pm 39$ & $0.96 \pm 0.07$ & $2.87 \pm 0.19$ & $0.12 \pm 0.03^{\mathrm{a}}$ \\
\hline
\end{tabular}

Results expressed as mean \pm SEM.

a Significantly different from the control group $(p<0.05)$

b $n$ refers to total number of pregnant rats used in these experiments

${ }^{c} n$ refers to number of fetuses examined for body and lung weights from each group of mothers, i. e. control, diabetic and insulin-treated diabetic groups

on day 1 after parturition and declining thereafter to the gestational level. The labelling of PC was not different in maternal lungs from the control, diabetic or insulintreated diabetic groups at any gestational period (Fig. 1a). However, the labelling of PC in fetal lung slices on day 18 of gestation was lower than that of the maternal lung slices, remaining low on day 20 but increasing on day 21 to a comparable rate. This rate was increased further to an extremely high level on day 1 post-partum and declined on day 2 of life (Fig. $1 \mathrm{~b}$ ). There was no significant difference in the choline incorporation into $\mathrm{PC}$ by lungs from the control, diabetic and insulin-treated diabetic groups of pregnant rats and their offspring (Fig. 1b).

Secretion of radiolabelled PC from the maternal lungs was relatively low on day 18 of gestation, it increased gradually to a peak level on day 21 , remained high on day 1 after birth, and declined on day 2 to the gestational level (Fig. 2a). Secretion of PC from maternal lungs was not impaired by diabetes throughout gestation. However, on days 1 and 2 after parturition, PC secretion from maternal lungs was lower in diabetic

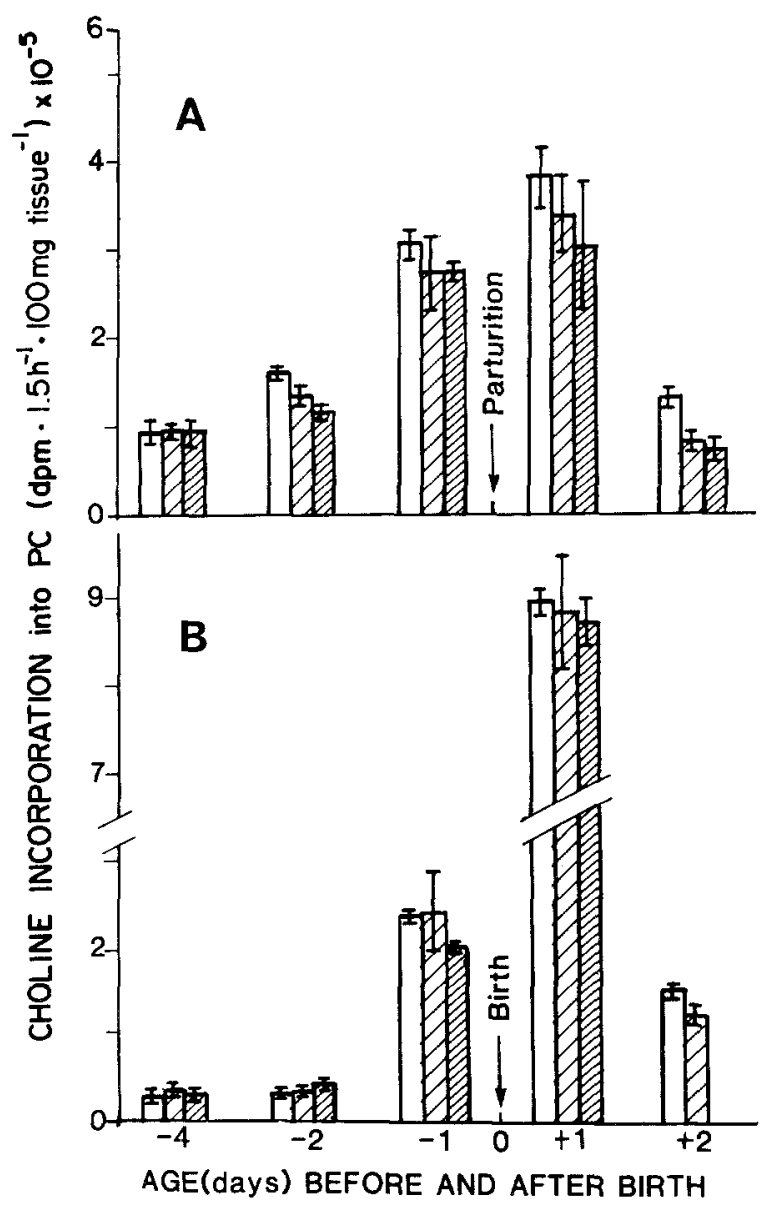

Fig. 1. Incorporation of (methyl- ${ }^{14} \mathrm{C}$ )choline into phosphatidyicholine (PC) of (A) maternal and (B) fetal/newborn rat lung during late gestation and the early neonatal period. Lung slices in triplicate sam-

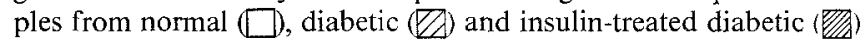
pregnant rats and their offspring were treated as described in Methods. The results are expressed as mean \pm SEM from three experiments

than in control animals. This decrease was abolished by insulin treatment for 1 week (Fig. 2 a).

Secretion of PC from fetal lungs on day 18 of gestation was almost one-half the rate of that from maternal lungs, remaining low throughout gestation, but increasing sixfold on day 1 of life, declining somewhat on day 2 postnatally (Fig. 2b). PC secretion from fetal lung of normal, diabetic and insulin-treated diabetic groups was similar during late gestation. However, PC secretion from the lungs of newborn of diabetic mothers was suppressed on day 1 of life compared with that from the lungs of fetuses of control mothers (Fig. 2b). Insulin treatment of the diabetic pregnant rats for 1 week restored PC secretion from the lungs of fetuses of diabetic mothers to the control values (Fig. 2b).

Serum phospholipids in pregnant rats were low on day 18 of gestation, they increased gradually to peak levels near term and declined after parturition (Fig. $3 \mathrm{a}$ ). Similar alterations in the serum phospholipid levels of diabetic pregnant rats were observed (Fig. 3 b).

Alterations in maternal serum phospholipids during late gestation were similar to changes in the rate of PC 


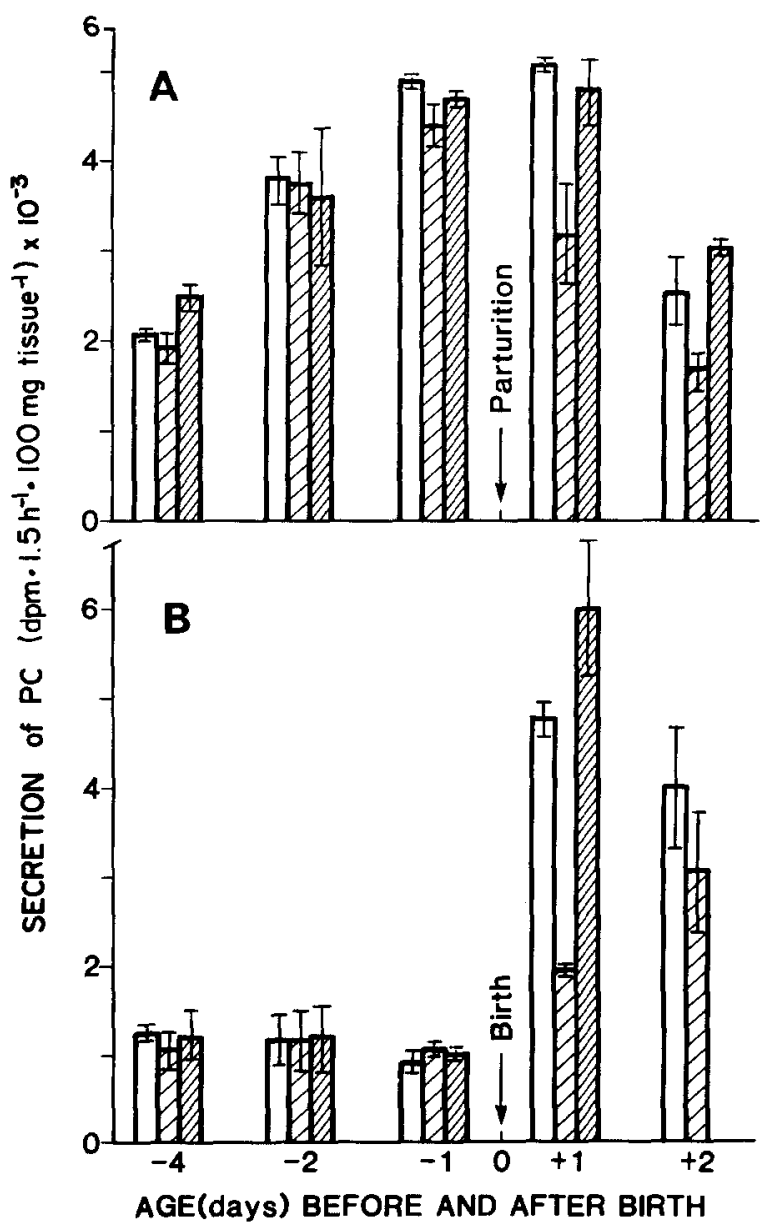

Fig. 2. Secretion of prelabelled phosphatidylcholine (PC) from (A) maternal and (B) fetal/newborn lungs. Lungs from normal $(\square)$, diabetic $(\square)$ and insulin-treated diabetic (ख) pregnant rats and their offspring during late gestation and the early neonatal period were used. The results are expressed as mean \pm SEM from four experiments

secretion from maternal lungs (Fig. $3 \mathrm{a}, \mathrm{b}$ ). However, an increase in PC synthesis by fetal lungs on day 21 followed an increase in PC secretion from maternal lungs and was accompanied by a concomittant decrease in maternal serum phospholipids (Fig. 3 a). PC secretion from the lungs of diabetic mothers was reduced in later stages of pregnancy and after parturition (Fig. 2a), although serum phospholipid levels in diabetic rats were similar to those in sera of the normal pregnant rats (Fig. $3 \mathrm{a}, \mathrm{b}$ ). Choline incorporation into PC of lungs of fetuses/newborn of diabetic mothers was not altered (Fig. 2a). These results suggest that there may be a relationship between maternal phospholipid metabolism and the synthesis of PC in lungs of fetuses/newborn of normal mothers and this relationship appears to be maintained during maternal diabetes.

\section{Discussion}

In the present study we utilized an experimental model, in which pregnant rats were made diabetic with streptozotocin, to examine the effects of maternal glucose in-
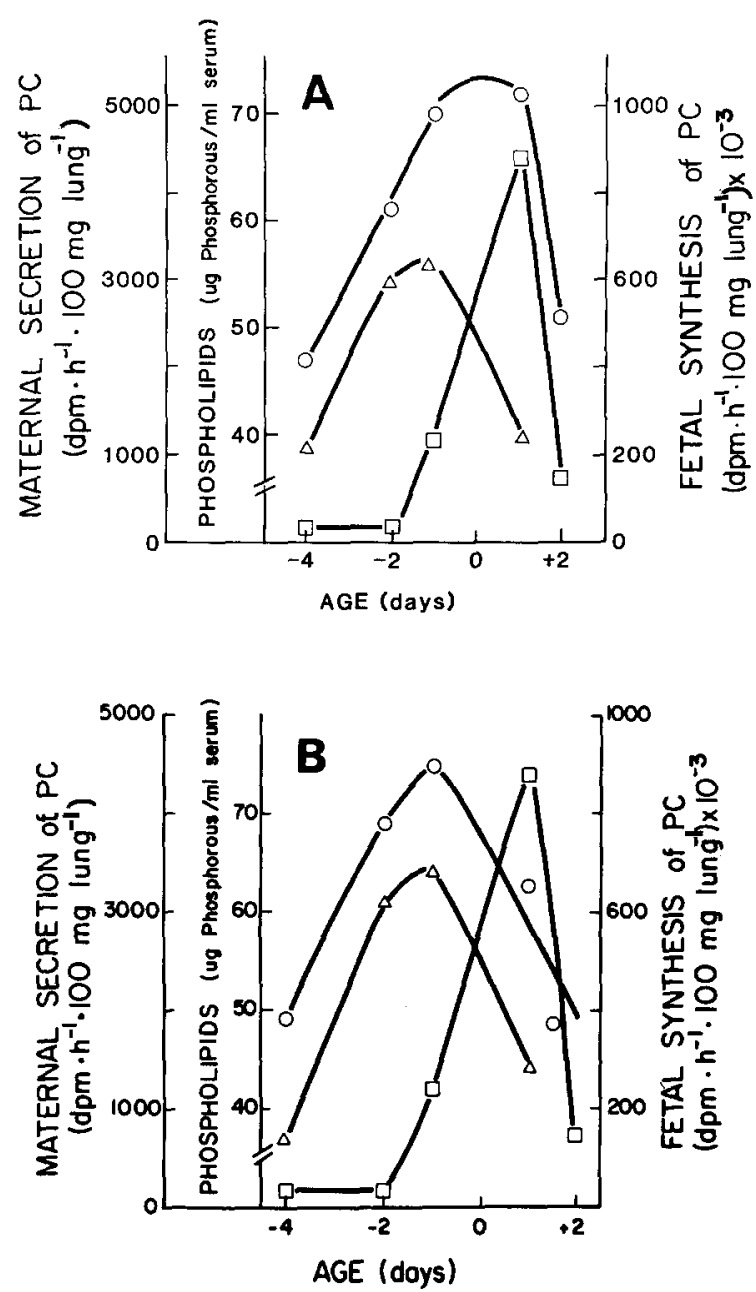

Fig. 3. Relationship between secretion of phosphatidylcholine (PC) from (A) normal and (B) diabetic maternal lungs $\left(\mathrm{O}_{-} \mathrm{O}\right.$ ), maternal serum phospholipids $(\triangle \longrightarrow \Delta)$ and $\mathrm{PC}$ synthesis in the lungs of respective fetuses $(\square-\square)$ during late gestation and the early neonatal period

tolerance on the synthesis and secretion of pulmonary $\mathrm{PC}$ in the mother, fetus and newborn. The results show that the incorporation of (methyl- ${ }^{14} \mathrm{C}$ ) choline into lung PC was not impaired in either the fetuses or the newborn of diabetic mothers, confirming previous observations $[16,17]$. Some investigators, however, have reported depressed PC synthesis in fetal lung of diabetic mothers $[14,15]$. The reason for this discrepancy is not understood but it may be related to the severity and duration of maternal diabetes [17, 34]. In some studies, acute effects of severe maternal diabetes on fetal lung development were investigated, whereas other studies examined the prolonged effects of mild and intermittent diabetes. The present findings that maternal diabetes in rats did not alter choline incorporation into lung $\mathrm{PC}$ of the offspring could result from multiple factors. Firstly, choline incorporation into $\mathrm{PC}$ of rat lung represents incorporation into total lung PC, including surfactant PC as well as membrane bound PC. Since surfactant PC constitutes a small percentage of the total PC in lungs, subtle changes in choline incorporation into surfactant PC may not be evident. However, this does not appear 
to be the case, since other investigators who studied choline incorporation into dipalmitoylphosphatidylcholine (DSPC), a true component of fetal lung surfactant, did not find any adverse effect of maternal diabetes on the synthesis of DSPC in fetal lungs $[16,17]$. Secondly, synthesis of PC in fetal lungs occurs through different pathways i.e. The cytidine $5^{\prime}$-diphosphocholine pathway, methylation of phosphatidylethanolamine to form PC, base-exchange and deacylation-acylation reaction mechanisms [10]. Although the cytidine 5'-diphosphocholine pathway accounts for about $90-95 \%$ of de novo synthesis of pulmonary PC in fetal lungs [35], it is possible that maternal diabetes affects some other pathway of PC synthesis in fetal lungs [15, 17]. Thirdly, another component of the surfactant i.e. phosphatidylglycerol is now considered to be important in lowering the surface tension of alveoli and this may be affected by maternal diabetes [36]. Finally, there could be an impairment of surfactant secretion from fetal lungs during maternal diabetes resulting in surfactant deficiency in the alveoli, despite adequate synthesis or storage of the surfactant in fetal lungs [37]. The present study demonstrates, for the first time, that maternal diabetes adversely affects secretion of PC from the lungs of offspring of diabetic mothers (Fig. 2b) and that the defective secretion of surfactant PC may be partly responsible for the respiratory distress syndrome in infants of diabetic mothers.

PC secretion from the lungs of fetuses of diabetic mothers was considerably suppressed on day 1 after birth compared with that from the lungs of fetuses of control pregnant rats (Fig. 2b). However, insulin treatment of the diabetic pregnant rats for 1 week restored $\mathrm{PC}$ secretion from the lungs of fetuses of diabetic mothers to control values (Fig. 2b). These results demonstrate that secretion of surfactant PC from the newborn rat lungs is dependent on the circulating levels of insulin and/or carbohydrate metabolism.

During maternal diabetes, the fetus is exposed to hyperglycaemia and hyperinsulinaemia [37-40], both of which inhibit glycogenolysis and lower synthesis of surfactant phospholipids in fetal lungs [9, 11, 12]. In the present study, rat fetuses were exposed to hyperglycaemia which subsided to almost normal serum glucose levels in newborn after parturition (Table 1). The results suggest that insulin secretion and its ability to lower blood glucose levels in newborns of diabetic mothers was not adversely affected. It is known that the offspring of diabetic mothers generally show macrosomia [40]. However, the body and lung weights of the fetuses of diabetic pregnant rats were little affected in the present study (Table 2). It is possible that the duration of maternal diabetes and exposure of rat fetuses to hyperglycaemia was not long enough to affect body and lung weights [34]. Further, it has been shown that mild and intermittent hyperglycaemia in pregnant mothers results in fetal macrosomia [37, 41], whereas severe hyperglycaemia, which appeared to be the case in the present study, brings about fetal microsomia [14, 17].
To examine a possible relationship between maternal phospholipid metabolism and fetal lung maturation, we examined the synthesis and secretion of PC in both fetal and maternal lungs together with maternal serum phospholipid levels on different days of gestation (Fig. $3 \mathrm{a}, \mathrm{b}$ ). The results show that alterations in maternal serum phospholipids during late pregnancy may reflect changes in the rate of PC secretion from maternal lungs. An increase in PC synthesis by fetal lungs on day 21 of gestation was accompanied by a concomittant decrease in the maternal serum phospholipids and followed the enhanced secretion of PC by the maternal lungs (Fig. $3 \mathrm{a}, \mathrm{b}$ ). These results suggest that there may be a dynamic relationship between maternal phospholipid metabolism, maternal serum phospholipid level and fetal lung maturation. Secretion of PC from lungs of diabetic pregnant rats was reduced in later stages of pregnancy and after parturition (Fig. 2 a). However, serum phospholipid levels in diabetic pregnant rats were not much affected (Fig. 3 a, b). These results are consistent with the view that there may be another tissue source of serum phospholipids in pregnant rats, such as the liver [42].

Glatz et al. [31] have shown the uptake of intact radiolabelled PC from the alveolus into lung tissue; the same PC was found later in the lamellar bodies. Biezenski [32] reported the placental transfer of intact phospholipids into fetal rabbit circulation during early gestation. However, near-term these phospholipids were partly stored in lung tissue and partly hydrolysed in the placenta, and the hydrolysed fragments appeared in fetal circulation and were utilized for the synthesis of phospholipids by fetal organs. Tsao et al. [33] reported enhanced synthesis and secretion of PC in lungs of pregnant rabbits treated with hydrocortisone. Since this treatment also resulted in enhanced synthesis of phospholipids by fetal lungs, these authors concluded that acceleration of PC secretion from maternal lungs by hydrocortisone may be related to enhanced fetal lung maturation in response to steroids. Shafrir and Khassis [34] have reported a significant correlation between the triglyceride and non-esterified fatty acid levels of the maternal plasma and the triglyceride levels of placenta and fetus in the case of rats made diabetic with streptozotocin on day 12 of pregnancy. These authors concluded that fetal lipids during rat pregnancy originate from both maternal fatty acids and those synthesized in situ, and the diabetes-induced increment in fetal triglyceride content is derived from pre-formed maternal triglyceride or non-esterified fatty acids. Results of all these studies appear to support the view that there may be a dynamic relationship between maternal phospholipid metabolism and fetal lung maturation during pregnancy.

Acknowledgements. This work was supported in parts by grants from the Medical Research Council of Canada (MT-6120) and the National Institutes of Health, USA (HL24515-03). L. Juravsky was supported by the Faculty of Medicine Research Fund. The authors appreciated 
the comments of L.H. Ryall, G. M. Hatch, E. Nadler and D. A. Howell on this manuscript.

\section{References}

1. Farrell PM, Avery ME (1975) Hyaline membrane disease. Am Rev Respir Dis 111: 657-658

2. Robert MF, Neff RK, Hubbell JP, Taeusch HW, Avery ME (1976) Association between maternal diabetes and the respiratory distress syndrome in the newborn. N Engl J Med 294: 357-360

3. Scholz RW, Woodward BM, Rhoades RA (1972) Utilization in vitro and in vivo of glucose and glycerol by rat lung. Am J Physiol 223: 991-996

4. Godinez RI, Longmore WJ (1973) Use of isolated perfused rat lung in studies on lung lipid metabolism. J Lipid Res 14: 138-144

5. Moxley MA, Longmore WJ (1977) Effect of experimental diabetes and insulin on lipid metabolism in the isolated perfused rat lung. Biochim Biophys Acta 488: 218-224

6. Maniscalco WM, Wilson CM, Gross I, Gobran L, Rooney SA, Warshaw JB (1978) Development of glycogen and phospholipid metabolism in fetal and newborn rat lung. Biochim Biophys Acta $530: 333-346$

7. Gilden C, Sevanian A, Tierney DF, Kaplan SA, Barrett CT (1977) Regulation of fetal lung phosphatidylcholine synthesis by cortisol: role of glycogen and glucose. Pediatr Res 11: 845-858

8. Benito M, Lorenzo M, Madina JM (1982) Relationship between lipogenesis and glycogen synthesis in maternal and foetal tissues during late gestation in the rat. Effect of dexamethasone. Biochem J 204: 865-868

9. Bourbon JR, Rieutort M, Engle MJ, Farrell PM (1982) Utilization of glycogen for phospholipid synthesis in fetal rat lung. Biochim Biophys Acta 712: 382-389

10. Van Golde LMG (1976) Metabolism of phospholipids in the lung. Am Rev Respir Dis 114: $977-1000$

11. Sanders RL, Longmore WJ (1975) Phosphatidylglycerol in rat lung. II. Comparison of occurrence, composition, and metabolism in surfactant and residual lung fractions. Biochemistry 14: $835-840$

12. Gross I, Smith GJW, Wilson CM, Maniscalco WM, Ingleson LD, Brehier A, Rooney SA (1980) The influence of hormones on the biochemical development of fetal rat lung in organ culture. II. Insulin. Pediatr Res 14: 834-838

13. Sosenko IRS, Frantz ID, Roberts RJ, Meyrick B (1980) Morphologic disturbance of lung maturation in fetuses of alloxan diabetic rabbits. Am Rev Respir Dis 122: 687-696

14. Rhoades RA, Filler DA, Vannata B (1979) Influence of maternal diabetes on lipid metabolism in neonatal rat lung. Biochim Biophys Acta 572: 132-138

15. Tyden O, Berne C, Eriksson U (1980) Lung maturation in fetuses of diabetic rats. Pediatr Res 14: 1192-1195

16. Tsai MY, Josephson MW, Brown DM (1981) Fetal rat lung phosphatidylcholine synthesis in diabetic and normal pregnancies. A comparison of prenatal dexamethasone treatments. Biochim Biophys Acta $664: 174-181$

17. Demottaz V, Epstein MF, Frantz ID (1980) Phospholipid synthesis in lung slices from fetuses of alloxan diabetic rabbits. Pediatr Res 14: 47-49

18. Ofulue AF, Nijjar MS (1981) Influence of streptozotocin-induced diabetes on the cytoplasmic factors modulating adenylate cyclase activity in rat lungs. Biochim Biophys Acta 675:397-402

19. Ofulue AF, Nijjar MS (1982) Effect of streptozotocin-induced diabetes on calmodulin and cyclic AMP phosphodiesterase activity in the rat lungs. Lung 160:303-310

20. Nijjar MS (1979) Regulation of rat lung adenylate cyclase by cytoplasmic factor(s) during development. Biochim Biophys Acta 584: $43-50$

21. Nijjar MS (1979) Role of cyclic AMP and related enzymes in rat lung growth and development. Biochim Biophys Acta 586: $464-472$

22. Nijjar MS, Thurlbeck WM (1980) Alteration in enzymes related to adenosine 3', 5'-monophosphate during compensatory growth of rat lung. Eur J Biochem 105: 403-407

23. Barrett CT, Sevanian A, Lavin N, Kaplan SA (1976) Role of adenosine 3',5'-monophosphate in maturation of fetal lungs. Pediatr Res 10:621-625

24. Stahlmann MT, Gray ME, Lieu S, Chytel F (1974) The role of cyclic AMP in lamellar body synthesis and secretion. Pediatr Res 8: 470

25. Sohn RJ, Mathe AA, Volicer L (1977) Role of cyclic nucleotides in the normal lung and in bronchial asthma. In: Volicer L (ed) Clinical aspects of cyclic nucleotides, Spectrum Publications, New York, pp 193-227

26. Nijjar MS, Khanura BS, Juravsky LI (1982) Effect of maternal diabetes on the secretion of surfactant from the fetal and maternal rat lungs in vitro. Am Rev Respir Dis 125: 198 (Abstract)

27. Huggett ASG, Nixon DA (1957) Enzymatic determination of blood glucose. Biochem J 66:12P

28. Folch J, Lees M, Sloane-Stanley GH (1957) A simple method for the isolation and purification of total lipids from animal tissues. J Biol Chem 226: 497-509

29. Mason KJ, Nellenbogen J, Clements JA (1976) Isolation of disaturated phosphatidylcholine with osmium tetroxide. J Lipid Res 17: 281-284

30. Dixon WJ, Massey FJ (1957) The mean estimation and tests of hypothesis. In: Dixon WJ, Massey FJ (eds) Introduction to statistical analysis. McGraw-Hill, New York, pp 112-138

31. Glatz T, Ikegami M, Jobe A (1982) Metabolism of exogenously administered natural surfactant in the newborn lamb. Pediatr Res 6: $711-715$

32. Biezenski JJ (1970) Role of placenta in fetal lipid metabolism. II. Phospholipid transfer in early rabbit gestation. Am J Obstet Gynecol 108: 638-642

33. Tsao FHC, Gutcher GR, Zachman RD (1979) Effect of hydrocortisone on the metabolism of phosphatidylcholine in maternal and fetal rabbit lungs and livers. Pediatr Res 13:997-1001

34. Shafrir E, Khassis S (1982) Maternal-fetal fat transport versus new fat synthesis in the pregnant diabetic rat. Diabetologia 22: 111-117

35. Epstein MF, Farrell PM (1975) The choline incorporation pathway: primary mechanism for de novo lecithin synthesis in fetal primate lung. Pediatr Res 9:658-665

36. Cunningham MD, Desai NS, Thompson SA, Green JM (1978) Amniotic fluid phosphatidylglycerol in diabetic pregnancies. Am J Obstet Gynecol 131: 719-724

37. Epstein MF, Farrell PM, Chez RA (1976) Fetal lung lecithin metabolism in the glucose-intolerant thesus monkey pregnancy. Pediatrics 57: 722-728

38. Obenshain SS, Adam PAJ, King KC, Teramo K, Raivio KO, Raiha N, Schwartz R (1970) Human fetal insulin response to sustained maternal hyperglycemia. N Engl J Med 283: 566-570

39. Cuezva JM, Burnett ES, Kerr DS, Rodman HM, Patel MS (1982) The newborn of diabetic rat. 1. Hormonal and metabolic changes in the postnatal period. Pediatr Res 16:632-637

40. Sosenko IR, Kitzmiller JL, Loo SW, Blix P, Rubenstein AH, Gabbay KH (1979) The infant of diabetic mother. Correlation of increased cord C-peptide levels with macrosomia and hypoglycemia. N Engl J Med 301: 859-862

41. Kim YS, Jatoi I, Kim Y (1980) Neonatal macrosomia in maternal diabetes. Diabetologia 18: 407-411

42. Fishler MC, Entenman C, Montgomery ML, Chaikoff IL (1943) The formation of phospholipid by the hepatectomized dog as measured with radioactive phosphorus. 1. The site of formation of plasma phospholipids. J Biol Chem 150:47-55

Received: 21 September 1983

and in revised form: 18 April 1984

Dr. M.S. Nijjar

Alvin Buckwold Centre Research Laboratory

Department of Pediatrics

University of Saskatchewan

Saskatoon, Saskatchewan

Canada S7N OX0 